\title{
Realidad matematizada, fuente inagotable del hacer matemática
}

\author{
Ing. Alba Vera ${ }^{15}$ \\ averavelez@gmail.com \\ Lcdo. Máximo Baque ${ }^{16}$ \\ maxwilbaq@hotmail.com
}

Recibido en noviembre de 2019, aceptado en diciembre de 2019

\section{Resumen}

Es innegable que la enseñanza de la matemática, caracterizada por la repetición mecánica, memorística, descontextualizada y abstracta, exige cambios urgentes; convencido de ello se dio inicio a un proceso de investigación con el propósito de posicionar una forma diferente de enseñar la matemática con una propuesta creativa, lúdica, realista e interdisciplinar. La investigación conllevó implementar un plan de acción para concienciar, desde la práctica y bajo la modalidad de Comunidad de Aprendizajes Mixtas (CAM), a un grupo de docentes, representantes de familias y estudiantes sobre la importancia de incorporar nuevas formas de enseñar matemática, que contribuyan a fomentar el desarrollo del pensamiento lógico-crítico, creativo, propositivo en los distintos actores educativos. Esta experiencia de CAM se llevó a cabo en los centros educativos Oswaldo Álvarez Barba, La Dolorosa y José de Anchieta por medio de la sensibilización, conformación e implementación de comunidades de aprendizaje en el área de matemática.

Palabras claves: matemática realista, enseñanza/aprendizaje, Comunidad de Aprendizajes Mixtas (CAM), pensamiento matemático lógico-crítico.

15 Ing. en alimentos por la Universidad Laica Eloy Alfaro de Manabí, Docente de matemática de la escuela de educación básica fiscomisional Fe y Alegría "Oswaldo Álvarez Barba" Manta, Manabí.

16 Lcdo. en Filosofía por la Pontificia Universitá Lateranense (Roma), Acompañante educativo en la Regional Manabí. Manta, Ecuador. 


\section{Abstract}

It is undeniable that the teaching of mathematics, characterized by mechanical, memoristic, decontextualized and abstract repetition, demands urgent changes; that is why, a research process was started, for the purpose of positioning a different way of teaching mathematics with a creative, playful, realistic and interdisciplinary proposal. The research led to implement an action plan that had to be built from practice to raise awareness, and under the Mixed Learning Community (CAM) modality, to a group of teachers, representatives of families and students about the importance of incorporating new ways of teaching mathematics. It contributes to encourage the development of logical-critical, creative, proactive thinking in the different educational actors. This CAM experience was carried out in Unidades Educativas Oswaldo Álvarez Barba, La Dolorosa and José de Anchieta by raising awareness, shaping and implementing learning communities in the area of mathematics.

Keywords: realistic mathemathics, teaching / learning, Mixed Learning Community (CAM), logical-critical mathematical thinking.

\section{Introducción}

Para comprender ciertas realidades de la cultura escolar es de gran importancia analizar las expresiones con las que los actores educativos -sobre todo docentes y estudiantes- se refieren a las asignaturas que se imparten, a las prácticas e incluso a los mismos actores; expresiones que, al repetirlas frecuentemente terminan por convertirse en creencias que son asumidas como "verdades". Algunos ejemplos: "el de matemática se la ha tomado conmigo", "la de sociales habla mucho", "el profesor no sabe", "ese es un vago, no pasará de año", "señores, en supletorio nos vemos"; a ello se añade las preocupaciones y exigencias que las familias tienen con sus representados en cuanto a calificaciones se refiere.

De todo ello, un caso muy llamativo -por los efectos causadosson las creencias que se han ido tejiendo en torno al área de matemática. 
Es bastante común considerar a esta área como la más difícil de todas las áreas de aprendizaje, tanto para los estudiantes -a quienes en su mayoría les resulta muy difícil de aprender- como para los docentes, a quienes en algunos casos les resulta compleja de explicar (por varias razones, entre ellas, que no tienen la pedagogía apropiada para la enseñanza o no han sido formados específicamente para enseñar esta especialidad). La tradición escolar nos ha heredado estas creencias como las más destacadas: "las matemáticas son sólo para inteligentes", "las mujeres no son buenas para las matemáticas", "la matemática es una ciencia exacta", "la matemática es una ciencia abstracta", "la matemática es un área muy compleja", "para entender la matemática hay que memorizar muchas reglas y fórmulas", "¿para qué sirven las matemáticas en la vida?", "voy a seguir sociales por no ver matemática", "las matemáticas son aburridas", "ese man17 de mate se cree el sábelo todo", la lista podría seguir. El punto es que estas expresiones a fuerza de repetirlas de generación en generación se han vuelto parte de la cultura escolar y han creado una mentalidad y predisposición inapropiada ante esta asignatura, condicionando sobremanera tanto el aprendizaje como la enseñanza.

Pero estas expresiones no son más que un reflejo, un modo de enunciar los problemas de fondo, yestos problemas son que la matemática en la tradición escolar ha sido concebida y aceptada como una ciencia abstracta sin más y no tanto lógica; siempre se la aborda desvinculada de la realidad y aislada de las otras áreas; el procedimiento que se sigue para la resolución de ejercicios matemáticos (confundiéndose esto con resolución de problemas) suele ser meramente mecánico, memorístico y repetitivo; quienes la imparten están tan convencidos que por ser una ciencia exacta el único recurso es aprender de memoria las fórmulas para aplicarlas tal cual porque es la única garantía inequívoca de resolver una incógnita; todo ello ha condicionado tanto la enseñanza como el aprendizaje, estancándose en lo mecánico, repetitivo y memorístico, descuidando enormemente el espíritu filosófico propio del pensamiento matemático y limitando de este modo el desarrollo del pensamiento reflexivo, lógico, crítico, propositivo, creativo e innovador.

17 Hombre en inglés; anglosajismo utilizado con mucha frecuencia entre los ecuatorianos, también se aplica a la forma femenina "esa man" 
Todas estas creencias que reflejan una realidad cifrada, son la cara opuesta al propósito que plantea el Ministerio de Educación ecuatoriano (MINEDUC) en el área de matemática cuando en la guía del docente afirma que "el conocimiento de la matemática fortalece la capacidad de razonar, abstraer, analizar, discrepar, decidir, sistematizar y resolver problemas. (...) Con bases matemáticas sólidas se da un aporte significativo en la formación de personas creativas, autónomas, comunicadoras y generadoras de nuevas ideas" (MINEDUC, 2016, p. 345). Por lo que no sólo es necesario tener claridad de estos nobles propósitos del currículo, ante todo urge instaurar nuevas metodologías en la enseñanza, de tal modo que el quehacer educativo en el aula en el día a día sea coherente, es más, sea una práctica concreta de lo que se busca como propósito; metodologías que promuevan el desarrollo del pensamiento lógico-matemático de forma crítica y se adapten a los diversos ritmos de aprendizaje; será necesario también que el diseño de "tareas" sea motivador para que los estudiantes planteen y resuelvan problemas de la vida cotidiana; incentivarlos a reinventar herramientas matemáticas a partir del contexto y situaciones reales; es decir, se necesita poner en práctica una matemática realista basada en la idea fundamental de Freudenthal (1991), para quien, "la matemática debe ser pensada como una actividad humana a la que todas las personas pueden acceder y la mejor forma de aprenderla es haciéndola" (citado por Bressan, Zolkower \& Gallego, 2004; v. digital, p. 73-74); y para ello es necesario seguir un proceso que él llama 'matematización de la realidad'.

Si la matemática surge como matematización (organización) de la realidad, el aprendizaje matemático debe originarse también en esa realidad. Esto no sólo significa mantener a esta disciplina conectada al mundo real o existente sino también a lo realizable, imaginable o razonable para los alumnos (lbíd. p.75)

Comulgando con estas ideas de Freudenthal, esta indagación pretende plantear bases para introducir cambios en la enseñanza del área de matemática, a partir de las vivencias experimentadas en espacios concretos, con estudiantes y docentes reales, en los centros educativos de Oswaldo Álvarez Barba y La Dolorosa de Fe y Alegría regional Manabí, ubicados en la ciudad de Manta (Ecuador); que a su 
vez busca ser un primer acercamiento y sintonía con el sentir y necesidad expresada de los estudiantes.

\section{Metodología}

En el ámbito educativo bien se podría optar por uno u otro método de investigación: cuantitativo de corte positivista, o cualitativo de corte social; sin embargo para nosotros educadores populares, por un lado, un referente innegable son los postulados de Paulo Freire, sobre todo en su obra Pedagogía del oprimido18, obra en que se sientan las bases para posicionar el diálogo y la horizontalidad como elementos irrenunciables para mantener una relación de igual a igual entre los "hombres del pueblo" y los investigadores, siendo un requisito no negociable, cuando de investigación se trata, establecer una participación auténtica entre investigadores y los sujetos de investigación; a toda costa se debe evitar una relación de sujeto-objeto, los supuestos 'investigados' son tan sujetos como 'los investigadores', y su participación e incidencia en la investigación es mucho más valiosa que la de los mismos investigadores; por otro lado, otro referente son las ideas de Orlando Fals-Borda, quien desde las ciencias sociales posiciona la idea de igualdad de condiciones entre el saber científico y el saber popular, incluso en el ámbito político. Teniendo como base estos referentes, adoptamos en esta investigación el método de Investigación-Acción-Participativa (IAP), puesto que se trata de lograr los cambios requeridos con y desde los actores directos, y no de un mero estudio de las problemáticas.

La IAP es un enfoque de investigación basado en el método científico de resolución de problemas, es decir, detectado el problema no se lo estudia como fenómeno sino que con la participación de los distintos involucrados se buscan las distintas alternativas de solución; la IAP, según Zuber-Skerritt (1992), como enfoque alternativo a la investigación social tradicional se caracteriza por ser práctica, participativa y colaborativa, emancipatoria, interpretativa y crítica (citado por Centro de Formación e Investigación, 2017, p. 27); por ello en el proceso de indagación se

18 Obra referente desde su 1ra edición en 1970, tanto que en el 2007 alcanzó la 18va reimpresión. 
priorizan espacios de reflexión donde participan en una relación de igual a igual los distintos actores: investigadores y sujetos actores del campo donde se realiza la investigación, en búsqueda conjunta de alternativas de soluciones ante el problema de interés común.

De allí que, en este caso, una vez determinado el problema central, el punto de partida es llevar a cabo un plan de IAP en el área de matemática (variable independiente: aula teorizada), el mismo que analizados los resultados, desembocará en un plan de acción (de la realidad matematizada al aula teorizada, por un aprendizaje significativo desde lo interdisciplinar), cuya intención es en lo posible, transformar las problemáticas detectadas en la praxis de aula. Mientras dure la ejecución del plan de acción, se observará con detenimiento el acontecer y reacciones frente a las propuestas de mejora, que a su vez serán sometidas a un nuevo ciclo de reflexión (para la mejora continua).

Para llevar a cabo esta investigación se consideró como campo de indagación los centros educativos de Fe y Alegría Regional Manabí19, cuatro centros educativos concretamente: tres en Manta (La Dolorosa Fe y Alegría 1, Oswaldo Álvarez Barba Fe y Alegría 2, U.E José de Anchieta Fe y Alegría 3) y uno en Portoviejo (Fe y Alegría Las Cumbres), cada uno en contextos diferentes. Se conformó el equipo de investigadores integrados por dos maestras y un maestro que por profesión se dedican a la enseñanza de esta ciencia y el coordinador pedagógico regional (cuatro en total).

Conformado el equipo y visualizado el problema central, en un espacio de deliberación se estableció: delimitar el campo de investigación entre los estudiantes de básica superior de los centros ya mencionados, hacer partícipe a todos los docentes posibles indistintamente del nivel en el que imparten clases e involucrar en la indagación la percepción de las madres/padres de familia.

En cuanto a las técnicas y diseño de instrumentos para

19 Al inicio de esta investigación se pensó también en la posibilidad de indagar en centros fiscales para que el trabajo de contraste sea mayor, pero al ampliarse demasiado el rango de intervención se desistió de la idea. 
la recolección de datos se optó por entrevistas con preguntas cuantitativas y cualitativas a docentes (16 preguntas), estudiantes (21 preguntas) y madres/padres de familias (8 preguntas); observaciones de clases y grupos focales. Para el diseño de estos instrumentos se establecieron categorías relacionadas a la problemática central, temáticas según actores (tanto para las entrevistas como para los grupos focales) e indicadores basados en las categorías para las observaciones de clases.

El total de actores involucrados y que participaron en todo este proceso se distribuyeron de la siguiente manera:

- En las entrevistas: 55 estudiantes de 8vo, 9no y 10mo, 15 docentes y 15 madres/padres de familia de los cuatro centros implicados.

- En la observación de clases: se observó a 13 docentes entre hombres y mujeres.

- Grupo focal: se realizó uno sólo (de cuatro previstos inicialmente), donde participaron 4 estudiantes (de 8vo, 9no y $10 \mathrm{mo}) 4$ madres/padres de familia, 3 docentes y 1 directivo.

Para la participación de todos estos actores no se estableció diferencia de género, primero porque no fue un enfoque considerado de manera explícita en esta investigación y, segundo porque se consideró más apropiado recoger el parecer de todos los actores posibles sin establecer ningún tipo de diferencias, puesto que todos estamos implicados como actores educativos, tanto en la enseñanza como en el aprendizaje.

El propósito de todo ello es claro: mejorar, no sólo la calidad de la práctica y el accionar humano, sino también la creación de comunidades educativas autocríticas y propositivas, mejorar las relaciones humanas (como proceso político) en el ámbito educativo cuya mayor expresión se da en la praxis en el aula (relación pedagógica en el aula); y comprobar en la práctica los nuevos aportes o enfoques teóricos. 


\section{Descripción de resultados de los datos recabados.}

¿Cuál es la postura, sentir y pensar de los actores consultados sobre la problemática detectada?, ¿qué ideas comunes fueron expresadas en los datos recogidos? A continuación, se explica, describe, analiza e interpreta en líneas generales, el sentir y pensar de los actores consultados; sentir y pensar recogidos por medio de las entrevistas (a estudiantes, madres/padres de familia, docentes) y observaciones de clase.

Los datos recabados, como ya se explicó en líneas anteriores, responden a intenciones tanto cuantitativa (objetivas) como cualitativas (subjetivas), pues de lo que se trata es hacer visible las percepciones subjetivas de los actores de cada una de las comunidades educativas de donde se tomó las muestras para esta indagación.

Un criterio de interpretación ha sido priorizar, del total de preguntas planteadas en las entrevistas, aquellas preguntas relacionadas de forma más directa con el problema detectado: clases expositivas de reglas y procedimientos matemáticos, complementadas con la realización de los ejemplos respectivos; dada la explicación, los estudiantes dedican el resto del tiempo de las horas de clases dentro del aula a resolver ejercicios matemáticos en los que deben aplicar mecánica y repetitivamente lo explicado en teoría; estas clases se completan con las tareas para la casa, que suelen ser una serie de más ejercicios matemáticos que deberán resolver los aprendices a fin de entrenarse a la perfección en lo "aprendido"; los ejercicios matemáticos, tanto los que deben ser resueltos en aula como los que quedan de tarea para resolver en casa, no pasan der ser formulaciones pensadas por la o el docente al puro estilo de combinaciones numéricas y alfabéticas, con incógnitas que despejar (o resolver), formulaciones que se van armando según se le vaya ocurriendo a la genialidad del docente, pero casi nunca están relacionadas o conectadas con situaciones o problemáticas reales por resolver o buscarle soluciones de forma creativa; práctica que al fomentar lo mecánico y memorístico limita el desarrollo de la creatividad y del pensamiento lógico-crítico-reflexivo.

Mientras que en el caso de las observaciones de clases se 
mantiene la totalidad de los resultados, debido a que este instrumento ayuda a visualizar en su conjunto aspectos relacionados directamente con el problema sobre el que se investiga.

De la entrevista realizada a estudiantes.

De un total de 22 preguntas realizadas a 55 estudiantes (entre hombres y mujeres) de la básica superior de cuatro centros educativos de Fe y Alegría regional Manabí, nos enfocamos sólo en seis de ellas cuyo propósito es evidenciar la opinión de estos actores referente a los aspectos que ellos aprecian o no de las matemáticas, al cómo reciben la enseñanza de esta ciencia, destacar lo más importante y proyectar el cómo le gustaría que sea la enseñanza de esta área. En la siguiente tabla (y en las posteriores) se podrá ver las preguntas planteadas, las opciones para elegir la respuesta, los cuatro centros educativos donde se aplicó los instrumentos, el porcentaje por opciones donde se resalta (en verde) los porcentajes más altos y la tendencia en cada pregunta (resaltado en amarillo)

\begin{tabular}{|c|c|c|c|c|c|c|}
\hline PREGUNTAS & OPCIONES & $\begin{array}{c}\text { LAS } \\
\text { CUMBRES }\end{array}$ & $\begin{array}{c}\text { OSWALDO } \\
\text { ÁLVAREZ }\end{array}$ & $\begin{array}{c}\text { LA } \\
\text { DOLOROSA }\end{array}$ & $\begin{array}{r}\text { JOSÉ DE } \\
\text { ANCHIETA }\end{array}$ & $\%$ \\
\hline \multirow{3}{*}{$\begin{array}{l}\text { 4. ¿Qué es lo que } \\
\text { te gusta de las } \\
\text { matemáticas? }\end{array}$} & Los problemas & $43 \%$ & $17 \%$ & $27 \%$ & $10 \%$ & $21 \%$ \\
\hline & $\begin{array}{l}\text { Los procedi- } \\
\text { mientos/ reglas. }\end{array}$ & $57 \%$ & $50 \%$ & $23 \%$ & $20 \%$ & $31 \%$ \\
\hline & La lógica & $0 \%$ & $33 \%$ & $50 \%$ & $70 \%$ & $48 \%$ \\
\hline \multirow{3}{*}{$\begin{array}{l}\text { 5. ¿Qué es lo que } \\
\text { no te gusta de las } \\
\text { matemáticas? }\end{array}$} & Los problemas & $29 \%$ & $69 \%$ & $60 \%$ & $45 \%$ & $53 \%$ \\
\hline & $\begin{array}{l}\text { Los procedi- } \\
\text { mientos/ reglas }\end{array}$ & $43 \%$ & $15 \%$ & $27 \%$ & $50 \%$ & $34 \%$ \\
\hline & La lógica & $29 \%$ & $15 \%$ & $13 \%$ & $5 \%$ & $13 \%$ \\
\hline \multirow{4}{*}{$\begin{array}{l}\text { 9. Cuando el } \\
\text { docente te da } \\
\text { una clase nueva } \\
\text { de matemática } \\
\text { ¿De qué manera } \\
\text { te explica todos } \\
\text { los procesos a } \\
\text { seguir? }\end{array}$} & Teórica & $33 \%$ & $37 \%$ & $22 \%$ & $29 \%$ & $28 \%$ \\
\hline & Lúdica & $0 \%$ & $0 \%$ & $25 \%$ & $6 \%$ & $12 \%$ \\
\hline & Práctica & $56 \%$ & $58 \%$ & $42 \%$ & $58 \%$ & $52 \%$ \\
\hline & Grupal & $11 \%$ & $5 \%$ & $11 \%$ & $6 \%$ & $8 \%$ \\
\hline
\end{tabular}




\begin{tabular}{|l|l|c|c|c|c|c|}
\hline \multirow{2}{*}{$\begin{array}{l}\text { 10. ¿Qué crees } \\
\text { que es más } \\
\text { importante en el } \\
\text { aprendizaje de la } \\
\text { matemática? }\end{array}$} & Las reglas & $14 \%$ & $53 \%$ & $29 \%$ & $21 \%$ & $30 \%$ \\
\cline { 2 - 7 } & Los procesos & $71 \%$ & $35 \%$ & $45 \%$ & $61 \%$ & $50 \%$ \\
\cline { 2 - 7 } & La lógica & $14 \%$ & $12 \%$ & $23 \%$ & $11 \%$ & $16 \%$ \\
\hline \multirow{2}{*}{$\begin{array}{l}19 . \\
\text { puede aprees que se } \\
\text { matemática por } \\
\text { medio del juego? }\end{array}$} & $\mathrm{Si}$ & $0 \%$ & $0 \%$ & $3 \%$ & $7 \%$ & $4 \%$ \\
\cline { 2 - 7 } & No & $100 \%$ & $100 \%$ & $88 \%$ & $100 \%$ & $96 \%$ \\
\hline \multirow{2}{20}{$\begin{array}{l}20 \\
\text { gustarímo te } \\
\text { sean las clases de } \\
\text { matemáticas? }\end{array}$} & Dinámicas & $50 \%$ & $38 \%$ & $58 \%$ & $61 \%$ & $54 \%$ \\
\cline { 2 - 7 } & Prácticas & $25 \%$ & $62 \%$ & $38 \%$ & $35 \%$ & $40 \%$ \\
\cline { 2 - 7 } & Teóricas & $25 \%$ & $0 \%$ & $4 \%$ & $4 \%$ & $6 \%$ \\
\hline
\end{tabular}

De la entrevista realizada a madres/padres de Familia.

Del total de 8 preguntas realizadas a 15 madres/padres de familia de cuatro centro educativos de Fe y Alegría regional Manabí, nos enfocamos sólo en seis de ellas cuyo propósito es poner en evidencia el parecer de estos actores con respecto a: la importancia que le otorgan al aprendizaje de matemática en la vida de su representado, la ayuda que brindan en casa en la resolución de problemas, dificultades con la que se encuentran y tipo de ayuda que solicitan a la hora de resolver problemas matemáticos en casa, cómo define la manera de enseñar matemática del docente y qué apreciación le dan sus representados a la matemática.

\begin{tabular}{|c|c|c|c|c|c|c|}
\hline PREGUNTAS (P) & OPCIONES (O) & $\begin{array}{c}\text { LAS } \\
\text { CUMBRES }\end{array}$ & $\begin{array}{c}\text { OSWALDO } \\
\text { ÁLVAREZ }\end{array}$ & $\begin{array}{c}\text { LA } \\
\text { DOLOROSA }\end{array}$ & $\begin{array}{r}\text { JOSÉ DE } \\
\text { ANCHIETA }\end{array}$ & $\%$ \\
\hline \multirow{2}{*}{$\begin{array}{l}\text { 1. ¿Cree usted que } \\
\text { las matemáticas son } \\
\text { importantes para el } \\
\text { aprendizaje de su } \\
\text { representado? }\end{array}$} & Si & $100 \%$ & $100 \%$ & $100 \%$ & $100 \%$ & $100 \%$ \\
\hline & No & $0 \%$ & $0 \%$ & $0 \%$ & $0 \%$ & $0 \%$ \\
\hline \multirow{4}{*}{$\begin{array}{l}\text { 2. Cuando su repre- } \\
\text { sentado realiza tareas } \\
\text { de matemática ¿con } \\
\text { la ayuda de quién las } \\
\text { realizas? }\end{array}$} & Solo & $0 \%$ & $40 \%$ & $33.3 \%$ & $60 \%$ & $40 \%$ \\
\hline & Mamá & $50 \%$ & $0 \%$ & $66.7 \%$ & $0 \%$ & $20 \%$ \\
\hline & Papá & $50 \%$ & $20 \%$ & $0 \%$ & $20 \%$ & $20 \%$ \\
\hline & Otros & $0 \%$ & $40 \%$ & $0 \%$ & $20 \%$ & $20 \%$ \\
\hline
\end{tabular}




\begin{tabular}{|c|c|c|c|c|c|c|}
\hline \multirow{8}{*}{$\begin{array}{l}\text { 3. ¿Qué tipo de dificul- } \\
\text { tades tiene Ud. para } \\
\text { ayudarle a resolver los } \\
\text { problemas de matemá- } \\
\text { tica a su representado? }\end{array}$} & No comprende & $40 \%$ & $0 \%$ & $40 \%$ & $0 \%$ & $20 \%$ \\
\hline & $\begin{array}{l}\text { No es bueno en } \\
\text { matemáticas }\end{array}$ & $0 \%$ & $40 \%$ & $40 \%$ & $0 \%$ & $20 \%$ \\
\hline & $\begin{array}{l}\text { El estudiante no } \\
\text { tiene claro }\end{array}$ & $20 \%$ & $20 \%$ & $0 \%$ & $0 \%$ & $10 \%$ \\
\hline & $\begin{array}{l}\text { Sin instrucción } \\
\text { primaria }\end{array}$ & $0 \%$ & $0 \%$ & $0 \%$ & $0 \%$ & $0 \%$ \\
\hline & $\begin{array}{l}\text { Los ejercicios } \\
\text { actuales no se } \\
\text { comprenden }\end{array}$ & $20 \%$ & $20 \%$ & $0 \%$ & $60 \%$ & $25 \%$ \\
\hline & No hay tiempo & $20 \%$ & $20 \%$ & $20 \%$ & $20 \%$ & $20 \%$ \\
\hline & $\begin{array}{l}\text { Todas las ante- } \\
\text { riores }\end{array}$ & $0 \%$ & $0 \%$ & $0 \%$ & $0 \%$ & $0 \%$ \\
\hline & Ninguna dificultad & $0 \%$ & $0 \%$ & $0 \%$ & $20 \%$ & $5 \%$ \\
\hline \multirow{4}{*}{$\begin{array}{l}\text { 4. Cuando usted no } \\
\text { puede ayudarle a } \\
\text { resolver un problema de } \\
\text { matemática a su repre- } \\
\text { sentado ¿cómo afronta } \\
\text { la situación? }\end{array}$} & Revisa el libro & $33.3 \%$ & $35 \%$ & $20 \%$ & $50 \%$ & $31.8 \%$ \\
\hline & $\begin{array}{l}\text { Pregunta al do- } \\
\text { cente }\end{array}$ & 0 & $37.5 \%$ & $60 \%$ & $16.7 \%$ & $31.8 \%$ \\
\hline & $\begin{array}{l}\text { Consulta en } \\
\text { internet }\end{array}$ & $33.3 \%$ & $12.5 \%$ & $20 \%$ & $3.33 \%$ & $22.8 \%$ \\
\hline & $\begin{array}{l}\text { Paga un tutor } \\
\text { particular }\end{array}$ & $33.3 \%$ & $25 \%$ & $0 \%$ & $0 \%$ & $13.6 \%$ \\
\hline \multirow{5}{*}{$\begin{array}{l}\text { 5. De qué manera } \\
\text { definiría el modo de } \\
\text { trabajar del docente de } \\
\text { matemática? }\end{array}$} & Tradicional & $0 \%$ & $0 \%$ & $0 \%$ & $0 \%$ & $0 \%$ \\
\hline & Creativo lúdico & $0 \%$ & $42.9 \%$ & $33.3 \%$ & $14.3 \%$ & $26.3 \%$ \\
\hline & Participativo & $0 \%$ & $28.6 \%$ & $66.7 \%$ & $42.9 \%$ & $36.8 \%$ \\
\hline & Cooperativo & $0 \%$ & $14.3 \%$ & $0 \%$ & $42.9 \%$ & $21.1 \%$ \\
\hline & No sé & $100 \%$ & $14.3 \%$ & $0 \%$ & $0 \%$ & $15.8 \%$ \\
\hline
\end{tabular}

De la entrevista realizada a Docentes.

De 16 preguntas planteadas a 15 docentes de cuatro centro educativos de Fe y Alegría regional Manabí, nos enfocamos sólo en cuatro de ellas (por razones antes expuestas: preguntas relacionadas de manera directa con el problema) cuyo propósito es poner en evidencia el parecer de estos actores con respecto a: el enfoque pedagógico y metodología desde donde enseñan matemática, el tiempo de su última actualización pedagógica a nivel de conocimientos, interés que muestran los padres de familias por el rendimiento de sus representados y la frecuencia con que plantean problemas que generan análisis lógicocrítico matemático aplicado a la realidad. 
Tabla $\mathbf{N}^{\circ}$ 3: Resultados entrevista a docentes

\begin{tabular}{|c|c|c|c|c|c|c|}
\hline PREGUNTAS (P) & $\begin{array}{l}\text { OPCIONES } \\
(0)\end{array}$ & $\begin{array}{c}\text { LAS } \\
\text { CUMBRES }\end{array}$ & $\begin{array}{c}\text { OSWALDO } \\
\text { ÁLVAREZ }\end{array}$ & $\begin{array}{c}\text { LA } \\
\text { DOLOROSA }\end{array}$ & $\begin{array}{l}\text { JOSÉ DE } \\
\text { ANCHIETA }\end{array}$ & $\%$ \\
\hline \multirow{5}{*}{$\begin{array}{l}\text { 1. ¿Desde qué enfoque } \\
\text { pedagógico y con qué } \\
\text { metodología enseña las } \\
\text { matemáticas? }\end{array}$} & Conductista & $0 \%$ & $0 \%$ & $0 \%$ & $0 \%$ & $0 \%$ \\
\hline & Cognitivista & $0 \%$ & $50 \%$ & $0 \%$ & $0 \%$ & $20 \%$ \\
\hline & $\begin{array}{l}\text { Constructi- } \\
\text { vista }\end{array}$ & $0 \%$ & $17 \%$ & $100 \%$ & $100 \%$ & $53 \%$ \\
\hline & Tradicional & $100 \%$ & $33 \%$ & $0 \%$ & $0 \%$ & $27 \%$ \\
\hline & Otros & $0 \%$ & $0 \%$ & $0 \%$ & $0 \%$ & $0 \%$ \\
\hline \multirow{4}{*}{$\begin{array}{l}\text { 3. ¿Cuándo y cuál fue tu } \\
\text { último curso de actualiza- } \\
\text { ción en el campo de las } \\
\text { matemáticas? }\end{array}$} & Actualmente & $0 \%$ & $0 \%$ & $0 \%$ & $40 \%$ & $14 \%$ \\
\hline & Hace un año & $0 \%$ & $0 \%$ & $0 \%$ & $20 \%$ & $7 \%$ \\
\hline & $\begin{array}{l}\text { Hace tres } \\
\text { años }\end{array}$ & $50 \%$ & $0 \%$ & $0 \%$ & $20 \%$ & $14 \%$ \\
\hline & Otros & $50 \%$ & $83 \%$ & $100 \%$ & $20 \%$ & $64 \%$ \\
\hline \multirow{4}{*}{$\begin{array}{l}\text { 5. ¿Con qué frecuencia } \\
\text { los padres de familia lo } \\
\text { visitan para informarse } \\
\text { del desempeño de su } \\
\text { representado? }\end{array}$} & $\begin{array}{l}\text { Una vez por } \\
\text { semana }\end{array}$ & $100 \%$ & $17 \%$ & $50 \%$ & $40 \%$ & $40 \%$ \\
\hline & $\begin{array}{l}\text { Solo en } \\
\text { reuniones }\end{array}$ & $50 \%$ & $67 \%$ & $100 \%$ & $0 \%$ & $47 \%$ \\
\hline & $\begin{array}{l}\text { No asisten al } \\
\text { centro }\end{array}$ & $0 \%$ & $0 \%$ & $0 \%$ & $40 \%$ & $0 \%$ \\
\hline & Otros & $0 \%$ & $0 \%$ & $50 \%$ & $20 \%$ & $13 \%$ \\
\hline \multirow{3}{*}{$\begin{array}{l}\text { 9. En clases, ¿con qué } \\
\text { frecuencia planteas } \\
\text { problemas que generan } \\
\text { un análisis lógico-crítico } \\
\text { matemático y aplicado a } \\
\text { la realidad? }\end{array}$} & Siempre & $100 \%$ & $83 \%$ & $50 \%$ & $40 \%$ & $71 \%$ \\
\hline & A veces & $0 \%$ & $0 \%$ & $50 \%$ & $60 \%$ & $29 \%$ \\
\hline & Nunca & $0 \%$ & $0 \%$ & $0 \%$ & $0 \%$ & $0 \%$ \\
\hline
\end{tabular}

\section{De las Observaciones de clases.}

El instrumento de Observación de clases está estructurado en 5 categorías (Estrategias de Aula, Iniciativas Innovadoras en el área, Diseño y uso de recursos didácticos, Habilidad para plantear y resolver problemas nivel de reflexión, análisis y lógica, y Relación aula contexto) que a su vez se desglosan en 32 indicadores a través de los cuales se busca observar el nivel de concreción (alto, medio, bajo, nulo) de lo planteado en el currículo nacional, en perspectiva del uso de metodologías activas y dominio del sistema metodológico propio de Fe y Alegría, definido en 
el Horizonte Pedagógico Pastoral y conocido como CORDIS ${ }^{20}$, método que busca concretar en el aula los principios de la Educación Popular.

Una de las categorías a la que se le dio mayor importancia en la observación de clases es la referente a la Relación aula contexto, la idea es verificar la tendencia en este aspecto decisivo al momento de encontrar una motivación por la cual empeñarse en el estudio de las matemáticas, su aplicación a la vida cotidiana, para sacar mejor provecho a la gran oportunidad de integrar un sinnúmero de recursos que brinda el contexto y dar a los estudiantes una razón fundamental para aprender matemática de forma dinámica (como lo demandan); los datos recabados en esta categoría en particular son de suma importancia, puesto que las falencias evidenciadas son el punto de partida de las iniciativas que revolucionaran la praxis actual ya que urge apostar por el aprendizaje de una matemática aplicada, realista.

Estas observaciones se las realizó con el propósito de contrastar y corroborar, por observación directa en aula, lo opinado por los distintos actores a través de las entrevistas, por ello más que observar el desempeño del docente lo que se observó fue el acontecer pedagógico en un período clase de matemática, la interacción que se establece entre los dos actores principales del aula: docente y estudiantes, de allí que algunos indicadores están referidos a las reacciones de los estudiantes ante las propuestas pedagógicas de su docente. Se observó un total de 13 clases (13 docentes) entre los distintos niveles de enseñanza en los cuatro centros educativos escogidos para esta indagación.

La tabla resume los resultados en porcentajes; la interpretación se la realiza analizando las posibles tendencias a nivel regional en cada categoría, según los datos resaltados (porcentajes más alto) en cada uno de los indicadores. Cabe señalar que la apreciación que refleja este instrumento es directamente la del observador, basado en lo que observó en clase sin previa preparación intencionada de la clase a observar.

20 Palabra que hace referencia al término latino de corazón (cor, cordis) y se forma juntando las iniciales de cada una de las categorías que definen los principios de la Educación Popular: Contextualización (CO), Revalorización de saberes y experiencias (R), Diálogo de saberes (D), Innovación transformadora, Sistematización y socialización (S) La metodología conlleva la intencionalidad de educar desde, con y el corazón. 
Tabla $\mathbf{N}^{\circ}$ 4: Resultados observación de clase

\begin{tabular}{|c|c|c|c|c|c|c|c|}
\hline CATEGORÍAS & INDICADORES & OPCIONES & $\begin{array}{c}\text { LAS } \\
\text { CUMBRES }\end{array}$ & $\begin{array}{c}\text { JOSE DE } \\
\text { ANCHIETA }\end{array}$ & $\begin{array}{c}\text { OSWALDO } \\
\text { ÁLVAREZ }\end{array}$ & $\begin{array}{c}\text { LA } \\
\text { DOLOROSA }\end{array}$ & $\%$ \\
\hline \multirow{24}{*}{$\begin{array}{l}\text { Estrategias de } \\
\text { aula }\end{array}$} & \multirow{4}{*}{$\begin{array}{l}\text { Aplicación de } \\
\text { estrategias } \\
\text { que permiten } \\
\text { salir de lo } \\
\text { tradicional }\end{array}$} & Alto & $0 \%$ & $0 \%$ & $33 \%$ & $0 \%$ & $9 \%$ \\
\hline & & Medio & $0 \%$ & $33 \%$ & $67 \%$ & $0 \%$ & $27 \%$ \\
\hline & & Bajo & $33 \%$ & $0 \%$ & $0 \%$ & $0 \%$ & $9 \%$ \\
\hline & & Nulo & $67 \%$ & $67 \%$ & $0 \%$ & $100 \%$ & $55 \%$ \\
\hline & \multirow{4}{*}{$\begin{array}{l}\text { Estrategias } \\
\text { lúdicas e } \\
\text { innovadoras }\end{array}$} & Alto & $0 \%$ & $0 \%$ & $33 \%$ & $0 \%$ & $9 \%$ \\
\hline & & Medio & $0 \%$ & $33 \%$ & $33 \%$ & $0 \%$ & $18 \%$ \\
\hline & & Bajo & $33 \%$ & $0 \%$ & $33 \%$ & $0 \%$ & $18 \%$ \\
\hline & & Nulo & $67 \%$ & $67 \%$ & $0 \%$ & $100 \%$ & $55 \%$ \\
\hline & \multirow{4}{*}{\begin{tabular}{|l} 
Estrategias \\
motivadoras
\end{tabular}} & Alto & $0 \%$ & $0 \%$ & $33 \%$ & $0 \%$ & $9 \%$ \\
\hline & & Medio & $0 \%$ & $33 \%$ & $67 \%$ & $0 \%$ & $27 \%$ \\
\hline & & Bajo & $100 \%$ & $0 \%$ & $0 \%$ & $0 \%$ & $27 \%$ \\
\hline & & Nulo & $0 \%$ & $67 \%$ & $0 \%$ & $100 \%$ & $36 \%$ \\
\hline & \multirow{4}{*}{$\begin{array}{l}\text { Estrategias } \\
\text { que fomentan } \\
\text { la participa- } \\
\text { ción }\end{array}$} & Alto & $0 \%$ & $0 \%$ & $67 \%$ & $0 \%$ & $18 \%$ \\
\hline & & Medio & $0 \%$ & $67 \%$ & $33 \%$ & $0 \%$ & $27 \%$ \\
\hline & & Bajo & $100 \%$ & $0 \%$ & $0 \%$ & $50 \%$ & $36 \%$ \\
\hline & & Nulo & $0 \%$ & $33 \%$ & $0 \%$ & $50 \%$ & $18 \%$ \\
\hline & \multirow{4}{*}{\begin{tabular}{|l} 
Interés de \\
ESTS. por las \\
estrategias
\end{tabular}} & Alto & $0 \%$ & $0 \%$ & $33 \%$ & $0 \%$ & $9 \%$ \\
\hline & & Medio & $33 \%$ & $33 \%$ & $33 \%$ & $0 \%$ & $27 \%$ \\
\hline & & Bajo & $67 \%$ & $0 \%$ & $33 \%$ & $100 \%$ & $45 \%$ \\
\hline & & Nulo & $0 \%$ & $67 \%$ & $0 \%$ & $0 \%$ & $18 \%$ \\
\hline & \multirow{4}{*}{$\begin{array}{l}\text { No aplicación } \\
\text { de estrategias }\end{array}$} & Alto & $67 \%$ & $33 \%$ & $0 \%$ & $0 \%$ & $27 \%$ \\
\hline & & Medio & $33 \%$ & $0 \%$ & $33 \%$ & $0 \%$ & $18 \%$ \\
\hline & & Bajo & $0 \%$ & $0 \%$ & $67 \%$ & $0 \%$ & $18 \%$ \\
\hline & & Nulo & $0 \%$ & $67 \%$ & $0 \%$ & $100 \%$ & $36 \%$ \\
\hline \multirow{4}{*}{$\begin{array}{l}\text { Iniciativas } \\
\text { innovadoras } \\
\text { en el área }\end{array}$} & \multirow{4}{*}{$\begin{array}{l}\text { Metodologías } \\
\text { diferentes al } \\
\text { ERCA }\end{array}$} & Alto & $0 \%$ & $0 \%$ & $67 \%$ & $0 \%$ & $18 \%$ \\
\hline & & Medio & $0 \%$ & $33 \%$ & $33 \%$ & $0 \%$ & $18 \%$ \\
\hline & & Bajo & $0 \%$ & $0 \%$ & $0 \%$ & $0 \%$ & $0 \%$ \\
\hline & & Nulo & $100 \%$ & $67 \%$ & $0 \%$ & $100 \%$ & $64 \%$ \\
\hline
\end{tabular}




\section{¿Qué apreciación general reflejan estos datos?}

Desde la percepción de los estudiantes: les gusta más la lógica (48\%) con respecto a los procedimientos (31\%) y los problemas (21\%); de hecho, los problemas matemáticos es lo que menos les gusta (53\%). Mientras que con respecto a los modos de enseñar se aprecia una percepción de teórico - práctico ( $28 \%$ y $52 \%$ respectivamente), pero entiéndase lo "práctico" no como aplicación de la matemática a resolución de problemas reales, sino resolución de ejercicios matemáticos uno tras de otro en el aula o como tareas para la casa. Sin embargo, al preguntar sobre lo más importante en la matemática el mayor porcentaje lo llevan los procesos (50\%) Mayoritariamente se cree que se puede aprender matemática de forma lúdica (96\%), ello se refleja también en la forma cómo les gustaría que se enseñara esta área: dinámica (54\%) y práctica (40\%), menos teórica (6\%). Datos que en definitiva reflejan que por parte de los estudiantes hay como un deseo latente de cambios en los modos de enseñar las matemáticas.

Desde el punto de vista de los representantes, padres/madres, definitivamente las matemáticas son de suma importante para la vida (100\%); sin embargo, no siempre se da la ayuda necesaria en casa: en un $40 \%$ los estudiantes trabajan solos, y otro tanto tiene la ayuda de los padres (papá, 20\%, mamá 20\%). Y esto se debe a una cierta variedad de problemáticas cuando de ayudar a resolver problemas matemáticos se trata: no se comprende (20\%), no se es bueno en matemática (20\%), no hay tiempo (20\%), los ejercicios actuales no se comprenden (25\%); problemáticas que por lo general se resuelven consultando el libro (31.8\%), preguntando al docente (31.8\%), y en porcentaje menor, consultando internet (22.8\%)

Curiosamente los representantes tienen la apreciación que la forma de enseñar de los docentes en parte es cooperativa (21.1\%), creativo lúdico (26.3\%) y más aún participativo $(36,8 \%)$, menos tradicional (0\%).

Mientras que de lado de los docentes hay una convicción de $53 \%$ que consideran que la manera de enseñar es constructivista, le sigue lo tradicional (27\%) y lo cognitivista (20\%); sin embargo, en temas 
de formación continua se aprecia en un $64 \%$ que los últimos cursos de actualización lo habían realizado hace más de tres años, en contraste del $7 \%$ que refleja la actualización de hace un año. Un dato que difiere con la percepción de los representantes es el interés sobre el rendimiento de sus representados, el mayor porcentaje es que sólo preguntan en reuniones (47\%) mientras que, según el parecer de los docentes encuestados, al preguntarse sobre la frecuencia con que plantean problemas que generan un análisis lógico-crítico matemático y aplicado a la realidad, el mayor porcentaje dice que siempre, 70\%; parecer que difiere con la percepción de los estudiantes.

Todos los datos reflejados en las entrevistas se contrastaron as u vez con lo observado en clases; aquí con respecto a la aplicación de estrategias en aula, estrategias que salen de lo tradicional, el resultado es que un $55 \%$ es nulo, porcentaje que coincide con la aplicación de estrategias lúdicas e innovadoras, nulo (55\%); de igual modo, la aplicación de estrategias innovadoras el nivel es nulo (36\%); estrategias que fomentan la participación, nivel bajo (36\%); interés de los estudiantes por las estrategias, un nivel bajo (45\%); mientras que la no aplicación de estrategia muestra un nivel nulo en $36 \%$. Todo ello se corrobora al observar en qué medida los docentes toman iniciativas innovadoras en esta área, es decir, aplican metodologías distintas al ERCA, el nivel observado es nulo en un $64 \%$. No cabe duda que ante tal panorama, urge introducir cambios en las formas de enseñar las matemáticas.

\section{De la concienciación a la acción: logros de lo actuado (Discusión).}

"La matemática sólo se aprende haciéndola" (H. Fruedenthal)

En base a los datos analizados, que en gran parte confirman las hipótesis planteadas desde el inicio, y convencidos que son factibles otros modos de hacer matemáticas, dado que hay variedad de experiencias como la de la Enseñanza de la Matemática Realista, que va ganando terreno en varios países, es necesario profundizar sobre los principios de este enfoque, y dar el paso de la matematización de la realidad en doble sentido, por un lado organizar la enseñanza de las matemáticas incorporando la riqueza de elementos que brinda el contexto; y por otro lado, organizar realidades desde la visión de contenidos matemáticos. 
En un contexto donde la tradición escolar tiene muchas fuerzas (lo memorístico, la repetición, lo mecánico) y donde ganan más las fuerzas de las costumbres no es tan sencillo introducir cambios que modifican el modus faciendi. Sin embargo, el reto está ahí, vencer los miedos y arriesgarse a "practicar una pedagogía transformadora" (Freire \& Shor, 2014) impregna de todo un sentido nuevo a la vocación educadora, pues de nada serviría si la toma de conciencia no lleva a una acción transformadora; aquí es donde comienza el plan de acción que vio nacer a la propuesta de posicionar el enfoque de la enseñanza de la matemática realista. La puesta en marcha de este plan de acción llevó a evidenciar varios logros:

- Los estudiantes tuvieron el espacio apropiado para expresar libremente su sentir sobre la forma tradicional con que se les enseña la matemática y el cómo les gustaría que les enseñen.

- El diálogo, la reflexión con los docentes les permitió ubicarse desde otra visión sobre la enseñanza de la matemática, concienciarse que es posible cambiar la práctica mecánica y memorística con la que se venía trabajando, por una que sea lógica, contextualizada con la realidad de los estudiantes, reflexiva y propositiva.

- Experimentar como un gran acierto que madres/padres de familia sean parte del proceso enseñanza/aprendizaje de la matemática de una manera más cercana y activa en la misma aula, y no como simples veedores del aprendizaje y desarrollo de sus representado.

- Conocer el sentir de los estudiantes sobre la materia y sus vivencias con los docentes de matemáticas, significó una gran oportunidad para clarificar y se más asertivos en las mejoras que urgen consolidar.

- Todo el proceso de investigación en sí mismo, en cada una de sus fases, significó tener mayor cercanía tanto con los estudiantes como con los padres de familia, sentir sus realidades y comprender su postura dentro del proceso de enseñanza de la matemática. 
- El mejor logro fue unificar esfuerzos entre todas las partes involucradas a través de la conformación de Comunidades de Aprendizaje Mixtas (CAM), llamadas así porque fueron conformadas por madres y padres de familia, estudiantes y docentes; su finalidad es mediar el aprendizaje de los estudiantes en comunidad.

- Un punto favorable de las Comunidades de Aprendizaje Mixtas (CAM) fue la oportunidad de los espacios de estudios en conjunto, planificación de clases y desarrollo de las mismas como espacios de convivencia más estrecha entre todos los miembros de la comunidad educativa.

A través de las CAM en los centros educativos Oswaldo Álvarez y La Dolorosa, se realizaron varias sesiones de análisis y estudio del currículo de educación actual, planificación de clases con metodología CORDIS y posteriormente, el desarrollo de lo planificado, más en el centro educativo Oswaldo Álvarez Barba.

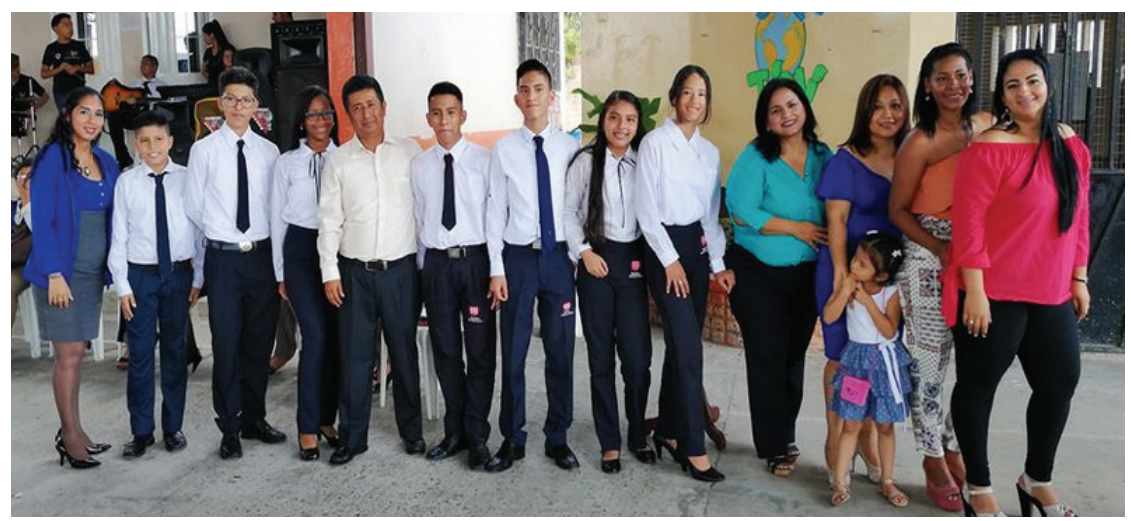

Pero también nos encontramos con algunas dificultades como la falta de compromiso de algunos padres con los encuentros de la comunidad de aprendizaje mixta; la estandarización de un sistema mecánico tradicionalista dentro del currículo actual que exige a los estudiantes estudiar bajo este sistema de tal modo que cuando llegan estudiantes de centros fiscales a centros educativos de Fe y Alegría les cuesta acoplarse a otras metodologías; todo ello llevó a ir modificando y adaptando lo proyectado según estos cambios de escenarios, por 
ejemplo se pasó a trabajar con CAM de décimo año de EGB en el centro educativo Oswaldo Álvarez Barba, ya no con novenos como inicialmente se tenía programado.

Independientemente de las adaptaciones que se fueron introduciendo para obtener mayor eficacia con las CAM, como es propio de la dinámica de toda experiencia nueva, compartir la experiencia de estudiar juntos el tema, planificar las clases y realizarla según lo planificado fue lo que más llenó de satisfacción a cada integrante, ver que el proyecto era viable; no sólo los estudiantes se divertían aprendiendo/ enseñando entre compañeros, sino también las madres/padres al asumir el rol de docente en aula experimentaban el gozo no sólo de compartir conocimientos, sino sobre todo de aprender en comunidad (lo de "comunidad de aprendizaje" se volvía vida, experiencia vital)

Por otro lado, la implementación del plan de acción favoreció a afianzar el deseo de cambiar la enseñanza de la matemática; al enseñar y estudiar la matemática de una forma lógica y experimental, esta se vuelve divertida y promueve el desarrollo del pensamiento matemático en los estudiantes.

\section{Conclusiones}

¿A qué conclusiones se puede llegar a partir de la interpretación de los resultados obtenidos en la indagación frente al problema planteado, en perspectiva de poner a disposición de la enseñanza un aporte que no signifique "agobio" para el docente sino al contrario, que reviva la pasión de una docencia comprometida en el cambio social?

Lo primero de todo es que los datos recabados permitieron corroborar algunas afirmaciones categóricas expresadas en el planteamiento del problema, según lo descrito en el análisis de los resultados: la enseñanza de la matemática está muy marcada por las formas tradicionales, privilegiando las facultades memorísticas, necesarias pero no únicas, y limitando el desarrollo del pensamiento matemático, lógico, crítico, creativo; con esta experiencia podemos concluir que la enseñanza de la matemática si puede ser activa y divertida. 
Finalmente, realizar este tipo de investigación socio-educativa representa algunas ventajas: observar y analizar la práctica permite comprenderla desde la confrontación con prácticas más exitosas; comprenderla significa encontrar los elementos enraizados en la tradición escolar que no son tan favorables al mejoramiento de la enseñanza; hacerse consciente de ello significa acoger el desafío de salir del encasillamiento afrontando los propios miedos para arriesgar a inventar y aplicar nuevas metodologías de enseñanza acordes con las demandas de los contextos actuales.

\section{Bibliografía}

Bressan, A., Zolkower, B. E. T. I. N. A., \& Gallego, M. F. (2004). Los principios de la educación matemática realista. Reflexiones teóricas para la educación matemática. Versión digital consultada en junio de 2018, en:

https://isfdajullon-Irj.infd.edu.ar/aula/archivos/repositorio/750/923/ DOC1-principios-de-educacion-matematica-realista.pdf

Centro de Formación e Investigación (2017), Curso de investigación. Módulo dos. El método IAP. Quito: CFI.

Fals Borda, O. (1976). Ciencia propia y colonialismo intelectual. Bogotá: Punta de lanza.

Fe y Alegría Ecuador (2016). Horizonte Pedagógico Pastoral. Quito: Fe y Alegría Ecuador.

Freire, P. $\left(2007^{18}\right)$. Pedagogía del oprimido. Madrid: Siglo XXI. Traducción de Jorge Mellado

Freire, P., \& Shor, I. (2014). Miedo y Osadía. La cotidianidad del docente que se arriesga a practicar una pedagogía transformadora. Buenos Aires: Siglo XXI.

Freudenthal, H. (2002). Revisiting Mathematics Education. London: KLUWER ACADEMIC PUBLISHERS.

Gravemeijer, KPE. y Terwel J. (2000) HANS FREUDENTHAL, un matemático en Didáctica y teoría curricular. J. Currículo Studies, 
vol. 32, n6, 777-796 (Traducción interna para el Grupo Patagónico de Didáctica de la Matemática a cargo de Norma Saggesse, Fernanda Gallego y Ana Bressan. 2004) V. digital consultada en noviembre de 2017 en: https://www.researchgate.net/ publication/241866337_HANS_FREUDENTHAL_un_matematico_ en_Didactica_y_teoria_curricular

MINEDUC, (2016) Educación General Básica. Currículo de los Niveles de Educación Obligatoria. Subnivel ELEMENTAL. Quito: MINEDUC. Consultado en: https://educacion.gob.ec/wp-content/uploads/ downloads/2019/09/EGB-Eelemental.pdf 